\title{
Multiple Sclerosis Slice Identification by Haar Wavelet Transform and Logistic Regression
}

\author{
Xueyan $\mathrm{Wu}^{1,2,3}$, Mason Lopez ${ }^{4, \text { a, * }}$ \\ ${ }^{1}$ Jiangsu Key Laboratory of Advanced Manufacturing Technology, Huaiyin, Jiangsu 223003, China \\ ${ }^{2}$ School of Computer Science and Technology, Nanjing Normal University, Nanjing, Jiangsu 210023, \\ China \\ ${ }^{3}$ State Key Laboratory of Digital Publishing Technology, Beijing 100871, China \\ ${ }^{4}$ University of British Columbia, Vancouver, British Columbia V6T1Z4, Canada \\ ”amason.lopez@ubc.cabxueyanwuu@gmail.com
}

Keywords: multiple sclerosis; slice identification; Haar wavelet transform; logistic regression

\begin{abstract}
Aim) Currently, scholars tend to use computer vision approaches to implement multiple sclerosis (MS) identification. (Method) In this study, we proposed a novel MS slice identification system, based on Haar wavelet transform, principal component analysis, and logistic regression. (Result) Simulation results showed the accuracies of our method using 2-level, 3-level, and 4-level decomposition are $83.25 \pm 1.62 \%, 89.72 \pm 1.18 \%$, and $87.65 \pm 1.79 \%$, respectively. (Conclusion) Our method with 3-level decomposition achieved the best.
\end{abstract}

\section{Background}

Multiple sclerosis (MS) is a progressive disease that affects both brain and spinal cord [1]. Early symptoms are composed of tingling, weakness, blurred version, numbness, etc. [2]. The traditional MRI scanning based identification may meet with the "normal-appearing white matter" problem. Hence, it is necessary to develop new methods to identify MS plaques.

Traditionally, scholars have proposed many computer vision methods [3-5] in identify abnormal brain diseases [6-20], including MS slice identification. For example, Zhou (2016) [21].used stationary wavelet entropy, combined with several machine learning methods. Zhan and Chen (2016) [22] used biorthogonal wavelet transform and logistic regression. Karaca (2017) [23] used convex combination of infinite kernels.

Nevertheless, those methods are too complicated, and their models are difficult to train. In this study, we presented a novel and simple system, which was based on Haar wavelet transform, principle component, and logistic regression.

\section{Materials and Methods}

Thirty-four multiple sclerosis patients and thirty-three healthy subjects were enrolled from China local hospitals from 2013. Experienced radiologists were instructed to select the slice with MS plaques from the 34 patients, and select corresponding slices from 33 healthy subjects. In total, we selected 141 slices from MS patients and 148 slices from healthy controls.

Our method contains three-stages. In the first stage, Haar wavelet [24] was chosen to transform the brain slices from spatial domain to wavelet domain [25-31]. The mother wavelet Haar wavelet $\lambda(b)$ is defined with the form of

$$
\lambda(b)=\left\{\begin{array}{cc}
+1 & 0 \leq b \leq 1 / 2 \\
-1 & 1 / 2 \leq b \leq 1 \\
0 & \text { otherwise }
\end{array}\right.
$$

Here $b$ denotes the spatial axis: either horizontal or vertical. The scaling function $\theta(b)$ is defined as: 


$$
\theta(b)=\left\{\begin{array}{cc}
1 & 0 \leq b<1 \\
0 & \text { otherwise }
\end{array}\right.
$$

We chose to use a two-level Haar wavelet transform.

In the second stage, principle component was used to reduce the wavelet coefficients to only cover the threshold (THR) of total variances. We here set THR to $95 \%$.

In the third stage, logistic regression (LR) [32] was employed to be the classifier. The logistic regression is simpler and easier to train than multilayer perceptron [33-36], probabilistic neural network [37], convolutional neural network [38], support vector machine [39-43], etc. In total, the diagram of our method is depicted in Figure 1.

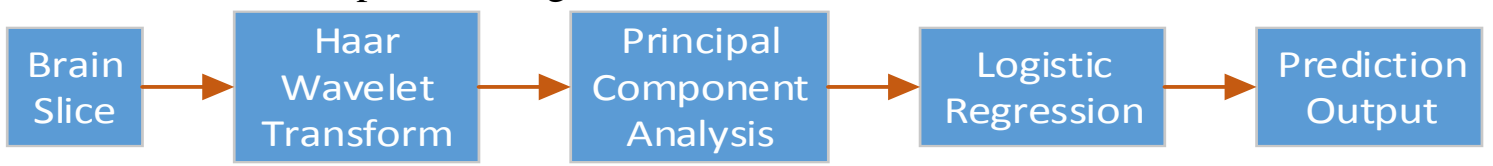

Figure 1. Diagram of proposed method

\section{Experiments Results and Discussions}

We measured the classified performance by the indicator of "accuracy", which is defined as the corrected identified number divided by the total sample number. Ten times of ten folds of cross validation was performed. The results were listed in Table 1, Table 2, and

Table 3. The accuracies of 2-level, 3-level, and 4-level decomposition are $83.25 \pm 1.62 \%$, $89.72 \pm 1.18 \%$, and $87.65 \pm 1.79 \%$, respectively. Hence, the 3 -level achieves the best performance.

Table 1. Accuracy of our method (2-level decomposition)

\begin{tabular}{|c|c|c|c|c|c|c|c|c|c|c|c|}
\hline Run & F1 & F2 & F3 & F4 & F5 & F6 & F7 & F8 & F9 & F10 & Total \\
\hline R1 & 86.21 & 90.00 & 79.31 & 86.21 & 86.21 & 82.76 & 89.66 & 82.14 & 82.76 & 85.71 & 85.12 \\
\hline R2 & 80.00 & 82.14 & 82.76 & 86.21 & 89.66 & 93.10 & 82.76 & 75.86 & 82.76 & 85.71 & 84.08 \\
\hline R3 & 79.31 & 93.10 & 82.14 & 93.33 & 75.86 & 86.21 & 89.66 & 79.31 & 78.57 & 86.21 & 84.43 \\
\hline R4 & 75.00 & 79.31 & 75.86 & 82.76 & 75.86 & 79.31 & 82.76 & 82.76 & 85.71 & 83.33 & 80.28 \\
\hline R5 & 79.31 & 82.14 & 93.10 & 89.66 & 89.66 & 82.14 & 83.33 & 82.76 & 86.21 & 82.76 & 85.12 \\
\hline R6 & 82.76 & 82.14 & 82.76 & 82.14 & 86.21 & 89.66 & 86.21 & 65.52 & 83.33 & 82.76 & 82.35 \\
\hline R7 & 86.21 & 89.66 & 79.31 & 86.21 & 75.86 & 85.71 & 82.76 & 86.21 & 79.31 & 82.76 & 83.39 \\
\hline R8 & 82.76 & 85.71 & 86.21 & 75.86 & 82.76 & 79.31 & 75.86 & 82.76 & 82.76 & 82.76 & 81.66 \\
\hline R9 & 79.31 & 86.21 & 85.71 & 89.29 & 82.76 & 79.31 & 86.21 & 86.67 & 82.76 & 82.76 & 84.08 \\
\hline R10 & 89.29 & 79.31 & 79.31 & 79.31 & 85.71 & 80.00 & 93.10 & 75.86 & 86.21 & 72.41 & 82.01 \\
\hline Avr & & & & & & & & & & & $83.25 \pm 1.62$ \\
\hline
\end{tabular}

Table 2. Accuracy of our method (3-level decomposition)

\begin{tabular}{|c|c|c|c|c|c|c|c|c|c|c|c|}
\hline Run & F1 & F2 & F3 & F4 & F5 & F6 & F7 & F8 & F9 & F10 & Total \\
\hline R1 & 96.55 & 86.21 & 96.55 & 96.43 & 96.67 & 86.21 & 86.21 & 89.29 & 96.55 & 75.86 & 90.66 \\
\hline R2 & 93.10 & 93.10 & 93.10 & 82.76 & 93.10 & 89.66 & 86.21 & 89.66 & 89.66 & 92.86 & 90.31 \\
\hline R3 & 86.21 & 86.21 & 89.66 & 90.00 & 93.10 & 89.29 & 93.10 & 86.21 & 89.66 & 89.29 & 89.27 \\
\hline R4 & 85.71 & 93.10 & 78.57 & 89.66 & 96.55 & 96.55 & 75.86 & 96.55 & 86.21 & 93.33 & 89.27 \\
\hline R5 & 93.10 & 93.10 & 96.43 & 89.66 & 89.66 & 89.66 & 89.66 & 89.66 & 93.10 & 93.10 & 91.70 \\
\hline R6 & 86.21 & 89.66 & 93.10 & 89.66 & 93.10 & 92.86 & 89.66 & 89.29 & 90.00 & 93.10 & 90.66 \\
\hline R7 & 89.66 & 85.71 & 89.66 & 89.66 & 86.21 & 82.76 & 86.21 & 89.66 & 93.10 & 89.66 & 88.24 \\
\hline R8 & 93.10 & 89.66 & 86.21 & 92.86 & 86.21 & 96.55 & 96.67 & 79.31 & 89.29 & 93.10 & 90.31 \\
\hline R9 & 82.76 & 82.14 & 89.66 & 86.21 & 82.76 & 89.66 & 93.10 & 93.10 & 93.10 & 89.66 & 88.24 \\
\hline R10 & 89.29 & 86.21 & 89.29 & 89.66 & 89.66 & 83.33 & 89.66 & 89.66 & 86.21 & 93.10 & 88.58 \\
\hline Avr & & & & & & & & & & & $89.72 \pm 1.18$ \\
\hline
\end{tabular}


Table 3. Accuracy of our method (4-level decomposition)

\begin{tabular}{|c|c|c|c|c|c|c|c|c|c|c|c|}
\hline Run & F1 & F2 & F3 & F4 & F5 & F6 & F7 & F8 & F9 & F10 & Total \\
\hline R1 & 82.76 & 86.21 & 89.66 & 82.14 & 80.00 & 89.66 & 89.66 & 85.71 & 89.66 & 89.66 & 86.51 \\
\hline R2 & 86.21 & 86.21 & 86.21 & 86.21 & 86.21 & 85.71 & 82.76 & 89.66 & 89.66 & 89.66 & 86.85 \\
\hline R3 & 82.76 & 85.71 & 89.66 & 89.29 & 90.00 & 86.21 & 86.21 & 79.31 & 79.31 & 86.21 & 85.47 \\
\hline R4 & 86.21 & 86.21 & 78.57 & 89.66 & 85.71 & 86.21 & 93.10 & 79.31 & 82.76 & 90.00 & 85.81 \\
\hline R5 & 93.10 & 78.57 & 89.66 & 86.21 & 92.86 & 86.21 & 86.67 & 93.10 & 86.21 & 89.66 & 88.24 \\
\hline R6 & 86.21 & 89.66 & 89.29 & 86.21 & 82.76 & 89.66 & 89.66 & 93.10 & 90.00 & 89.29 & 88.58 \\
\hline R7 & 86.21 & 93.10 & 93.10 & 89.29 & 82.76 & 85.71 & 86.21 & 93.10 & 90.00 & 89.66 & 88.93 \\
\hline R8 & 89.29 & 93.10 & 89.29 & 86.21 & 93.10 & 93.10 & 86.21 & 89.66 & 89.66 & 93.33 & 90.31 \\
\hline R9 & 85.71 & 86.21 & 86.21 & 89.66 & 89.66 & 83.33 & 86.21 & 85.71 & 79.31 & 86.21 & 85.81 \\
\hline R10 & 93.10 & 86.21 & 89.29 & 89.66 & 86.67 & 86.21 & 93.10 & 96.55 & 89.66 & 89.29 & 89.97 \\
\hline Avr & & & & & & & & & & & $87.65 \pm 1.79$ \\
\hline
\end{tabular}

\section{Conclusion and Future Research}

In this study, our team presented a novel multiple-sclerosis slice identification method, based on Haar wavelet transform, principal component analysis, and logistic regression. The simulation results showed 3-level Haar decomposition performed the best.

\section{Acknowledgments}

The paper is supported by Open fund for Jiangsu Key Laboratory of Advanced Manufacturing Technology (HGAMTL1601).

\section{References}

[1]. Nafisi-Moghadam, R., A. Rahimdel, T. Shanbehzadeh, et al., Comparison of Diffuse Weighted Imaging and Fluid Attenuation Inversion Recovery Sequences of MRI in Brain Multiple Sclerosis Plaques Detection. Iranian Journal Of Child Neurology, 2017. 11 (1): pp. 13-20

[2]. Sanchez, A.G., E.L. Andrade, J.V. Marsal, et al., A study to evaluate the effect of ultrasound treatment on nodules in multiple sclerosis patients. The International journal of neuroscience, 2017. 127 (5): pp. 404-411

[3]. Zhang, Y.D., Comment on 'Principles, developments and applications of computer vision for external quality inspection of fruits and vegetables: A review (Food Research International; 2014, 62: 326-343)'. Food Research International, 2015. 70: pp. 142-142

[4]. Ji, G., Fruit classification using computer vision and feedforward neural network. Journal of Food Engineering, 2014. 143: pp. 167-177

[5]. Wu, L., Classification of fruits using computer vision and a multiclass support vector machine. Sensors, 2012. 12 (9): pp. 12489-12505

[6]. Chen, Y., A Feature-Free 30-Disease Pathological Brain Detection System by Linear Regression Classifier. CNS \& Neurological Disorders - Drug Targets, 2017. 16 (1): pp. 5-10

[7]. Lu, S. and Z. Lu A pathological brain detection system based on kernel based ELM. Multimedia Tools and Applications, 2016, DOI: 10.1007/s11042-016-3559-z (Online).

[8]. Zhou, X.-X., J.-F. Yang, H. Sheng, et al., Combination of stationary wavelet transform and kernel support vector machines for pathological brain detection. Simulation, 2016. 92 (9): pp. $827-837$ 
[9]. Atangana, A. Application of stationary wavelet entropy in pathological brain detection. Multimedia Tools and Applications, 2016, DOI: 10.1007/s11042-016-3401-7 (Online).

[10]. Lu, Z., A Pathological Brain Detection System Based on Radial Basis Function Neural Network. Journal of Medical Imaging and Health Informatics, 2016. 6 (5): pp. 1218-1222

[11]. Lu, S. and X. Qiu, A Pathological Brain Detection System based on Extreme Learning Machine Optimized by Bat Algorithm. CNS \& Neurological Disorders - Drug Targets, 2017. 16 (1): pp. 23-29

[12]. Chen, X.-Q., Fractal dimension estimation for developing pathological brain detection system based on Minkowski-Bouligand method. IEEE Access, 2016. 4: pp. 5937-5947

[13]. Yang, M., Dual-Tree Complex Wavelet Transform and Twin Support Vector Machine for Pathological Brain Detection. Applied Sciences, 2016. 6 (6), Article ID: 169

[14]. Yang, J., Pathological brain detection in MRI scanning via Hu moment invariants and machine learning. Journal of Experimental \& Theoretical Artificial Intelligence, 2017. 29 (2): pp. 299-312

[15]. Sun, P., Preliminary research on abnormal brain detection by wavelet-energy and quantum-behaved PSO. Technology and Health Care, 2016. 24 (s2): pp. S641-S649

[16]. Sun, Y., A Multilayer Perceptron Based Smart Pathological Brain Detection System by Fractional Fourier Entropy. Journal of Medical Systems, 2016. 40 (7), Article ID: 173

[17]. Liu, G., Pathological brain detection in MRI scanning by wavelet packet Tsallis entropy and fuzzy support vector machine. SpringerPlus, 2015. 4 (1), Article ID: 716

[18]. Yang, X., P. Sun, Z. Dong, et al., Pathological Brain Detection by a Novel Image Feature-Fractional Fourier Entropy. Entropy, 2015. 17 (12): pp. 8278-8296

[19]. Feng, C., Feed-forward neural network optimized by hybridization of PSO and ABC for abnormal brain detection. International Journal of Imaging Systems and Technology, 2015. 25 (2): pp. 153-164

[20]. Sun, P., Pathological brain detection based on wavelet entropy and Hu moment invariants. Bio-Medical Materials and Engineering, 2015. 26 (s1): pp. 1283-1290

[21]. Zhou, X.-X., Comparison of machine learning methods for stationary wavelet entropy-based multiple sclerosis detection: decision tree, k-nearest neighbors, and support vector machine. Simulation, 2016.92 (9): pp. 861-871

[22]. Zhan, T.M. and Y. Chen, Multiple Sclerosis Detection Based on Biorthogonal Wavelet Transform, RBF Kernel Principal Component Analysis, and Logistic Regression. IEEE Access, 2016. 4: pp. 7567-7576

[23]. Karaca, Y., The Differential Diagnosis of Multiple Sclerosis Using Convex Combination of Infinite Kernels. CNS \& Neurological Disorders - Drug Targets, 2017. 16 (1): pp. 36-43

[24]. Ezzati, R. and S.M. Sadatrasoul, On numerical solution of two-dimensional nonlinear Urysohn fuzzy integral equations based on fuzzy Haar wavelets. Fuzzy Sets And Systems, 2017. 309: pp. 145-164

[25]. Yu, D., H. Shui, L. Gen, et al., Exponential wavelet iterative shrinkage thresholding algorithm with random shift for compressed sensing magnetic resonance imaging. IEEJ Transactions on Electrical and Electronic Engineering, 2015. 10 (1): pp. 116-117 
[26]. Zhang, Y.D., Z.C. Dong, G.L. Ji, et al., An improved reconstruction method for CS-MRI based on exponential wavelet transform and iterative shrinkagelthresholding algorithm. Journal of Electromagnetic Waves and Applications, 2014. 28 (18): pp. 2327-2338

[27]. Yang, J., Preclinical diagnosis of magnetic resonance (MR) brain images via discrete wavelet packet transform with Tsallis entropy and generalized eigenvalue proximal support vector machine (GEPSVM). Entropy, 2015. 17 (4): pp. 1795-1813

[28]. Chen, M., Y. Li, and L. Han, Detection of dendritic spines using wavelet-based conditional symmetric analysis and regularized morphological shared-weight neural networks. Computational and Mathematical Methods in Medicine, 2015, Article ID: 454076

[29]. Liu, A., Magnetic resonance brain image classification via stationary wavelet transform and generalized eigenvalue proximal support vector machine. Journal of Medical Imaging and Health Informatics, 2015. 5 (7): pp. 1395-1403

[30]. Yang, J., Identification of green, Oolong and black teas in China via wavelet packet entropy and fuzzy support vector machine. Entropy, 2015. 17 (10): pp. 6663-6682

[31]. Wei, L., Fruit classification by wavelet-entropy and feedforward neural network trained by fitness-scaled chaotic ABC and biogeography-based optimization. Entropy, 2015. 17 (8): pp. 5711-5728

[32]. Mansourian, S., E.I. Darbandi, M.H.R. Mohassel, et al., Comparison of artificial neural networks and logistic regression as potential methods for predicting weed populations on dryland chickpea and winter wheat fields of Kurdistan province, Iran. Crop Protection, 2017. 93: pp. 43-51

[33]. Wang, S.-H. Single slice based detection for Alzheimer's disease via wavelet entropy and multilayer perceptron trained by biogeography-based optimization. Multimedia Tools and Applications, 2016, DOI: 10.1007/s11042-016-4222-4 (Online).

[34]. Zhang, Y., Stock market prediction of S\&P 500 via combination of improved BCO approach and BP neural network. Expert systems with applications, 2009. 36 (5): pp. 8849-8854

[35]. Wu, L., Weights optimization of neural network via improved BCO approach. Progress in Electromagnetics Research, 2008. 83: pp. 185-198

[36]. Wu, L., Crop Classification by forward neural network with adaptive chaotic particle swarm optimization. Sensors, 2011. 11 (5): pp. 4721-4743

[37]. Naggaz, N. and G. Wei, Remote-sensing Image Classification Based on an Improved Probabilistic Neural Network. Sensors, 2009. 9 (9): pp. 7516-7539

[38]. Acquarelli, J., T. van Laarhoven, J. Gerretzen, et al., Convolutional neural networks for vibrational spectroscopic data analysis. Analytica Chimica Acta, 2017. 954: pp. 22-31

[39]. Chen, M., Morphological analysis of dendrites and spines by hybridization of ridge detection with twin support vector machine. PeerJ, 2016. 4, Article ID: e2207

[40]. Gorriz, J.M. and J. Ramírez, Wavelet entropy and directed acyclic graph support vector machine for detection of patients with unilateral hearing loss in MRI scanning. Frontiers in Computational Neuroscience, 2016. 10, Article ID: 160

[41]. Dong, Z., Classification of Alzheimer disease based on structural magnetic resonance imaging by kernel support vector machine decision tree. Progress In Electromagnetics Research, 2014. 144: pp. 171-184 
[42]. 42. Wu, L., An MR brain images classifier via principal component analysis and kernel support vector machine. Progress In Electromagnetics Research, 2012. 130: pp. 369-388

[43]. Chen, S., J.-F. Yang, and P. Phillips, Magnetic resonance brain image classification based on weighted-type fractional Fourier transform and nonparallel support vector machine. International Journal of Imaging Systems and Technology, 2015. 25 (4): pp. 317-327 